Pacific Journal of Mathematic 


\title{
ON RELATIVELY BOUNDED PERTURBATIONS OF ORDINARY DIFFERENTIAL OPERATORS
}

\author{
Colin ClaRK
}

\begin{abstract}
This paper studies ordinary differential operators of the form

$$
(-1)^{m} D^{2 m}+Q_{2 m-1} D^{2 m-1}+\cdots+Q_{0},
$$

over a finite interval $I$. The coefficients $Q_{j}$ are bounded operators in $L_{2}(I)$. This operator is treated as a perturbation $T+A$ of the operator $T$, which is generated by the leading term $(-1)^{m} D^{2 m}$ plus suitable boundary conditions. The main hypothesis is that $Q_{2 m-1}$ can be written as the sum of a compact operator and a bounded operator of sufficiently small norm. Given that $T$ is a discrete spectral operator, with eigenvalues $\left\{\lambda_{n}\right\}$, it is shown that $T+A$ is also a discrete spectral operator, with eigenvalues $\left\{\lambda_{n}^{\prime}\right\}$ satisfying $\left|\lambda_{n}^{\prime}-\lambda_{n}\right|=O\left(\left|\lambda_{n}\right|^{k / 2 m}\right)$, where $k$ is the largest integer $\leqq 2 m-1$ for which $Q_{k} \neq 0$. Proofs are based on the method of contour integration of resolvent operators.
\end{abstract}

If $A$ and $T$ are given, closed operators in a Hilbert space $\mathfrak{S}$, with $\mathfrak{D}(A) \supset \mathfrak{D}(T)$, we say that $A$ is bounded relative to $T$ if there are constants $c_{1}, c_{2}$ such that

$$
\|A u\| \leqq c_{1}\|T u\|+c_{2}\|u\|, \quad(u \in \mathfrak{D}(T)) .
$$

The infimum of numbers $c_{1}$ such that (1.1) holds for some $c_{2}$ is called the T-bound of $A,|A|_{T}$. If $|A|_{T}=0$, then for any $\varepsilon>0$ one can find a constant $C_{\varepsilon}$ such that

$$
\|A u\| \leqq \varepsilon\|T u\|+C_{\varepsilon}\|u\|, \quad(u \in \mathfrak{D}(T)) .
$$

Operators $A$ and $T$ with $|A|_{T}=0$ arise in the theory of differential operators, both ordinary and partial of elliptic type, $T$ being generated by the highest order derivative terms, and $A$ by the lower order terms.

In this paper we consider differential operators of the form

$$
(-1)^{m} D^{2 m}+\sum_{j=0}^{2 m-1} Q_{j} D^{j} \quad(D=d / d x)
$$

over a finite interval $I$. The $Q_{k}$ are bounded operators in $L_{2}(I)$; with the exception of $Q_{2 m-1}$, they can be completely arbitrary. The operator (1.3) is treated as a perturbation of an operator $T$ generated by the leading term $(-1)^{m} D^{2 m}$ together with suitable boundary conditions; $T$ will be assumed to be a spectral operator in the sense of Dunford. 
(See Kramer [6] and Dunford-Schwartz [2, Part III] for classification of boundary conditions under which $(-1)^{m} D^{2 m}$ becomes spectral.) The perturbing operator $A$, given by

$$
A u=\sum_{j=0}^{2 m-1} Q_{j} D^{j} u \quad(u \in \mathfrak{D}(T)),
$$

is bounded relative to $T$ and satisfies (1.2) with

$$
C_{\varepsilon}=O\left(\varepsilon^{-k /(2 m-k)}\right) \quad(\varepsilon \rightarrow 0),
$$

where the integer $k$ is defined by

$$
Q_{k+1}=Q_{k+2}=\cdots=Q_{2 m-1}=0, \quad Q_{k} \neq 0 .
$$

Now suppose that the coefficient $Q_{2 m-1}$ can be written in the form

$$
Q_{2 m-1}=B_{1}+B_{2}
$$

where $B_{1}$ is a bounded operator of sufficiently small norm, and $B_{2}$ is a compact operator. Under certain mild hypotheses about the eigenvalues of $T$, we will show that then

(1) The eigenvalues $\lambda_{j}^{\prime}$ of $T+A$ are related to the eigenvalues $\lambda_{j}$ of $T$ by

$$
\left|\lambda_{j}^{\prime}-\lambda_{j}\right|=O\left(\left|\lambda_{j}\right|^{k / 2 m}\right) \quad(j \rightarrow \infty)
$$

where $k$ is determined by (1.6), and

(2) $T+A$ is a spectral operator.

The first of these results seems to be new; the second has been obtained recently by R.E.L. Turner [11]. Special cases were treated by J. Schwartz [9] and H. P. Kramer [6]. Our method is a natural extension of the method used by Schwartz; it differs considerably from the method of Kramer, and bears virtually no resemblance to that of Turner. What we do is to construct a family of disjoint circles $\left\{C_{j}\right\}$ in the complex plane, centered at the original eigenvalues $\lambda_{j}$ (for large $j$ ), and such that each $C_{j}$ also contains exactly one eigenvalue $\lambda_{j}^{\prime}$. We therefore have the formula

$$
E_{j}^{\prime}-E_{j}=\frac{1}{2 \pi i} \int_{C_{j}}\left[R_{\lambda}(T+A)-R_{\lambda}(T)\right] d \lambda
$$

for the spectral projections $E_{j}^{\prime}$ and $E_{j}$ of $T+A$ and $T$ respectively, corresponding to the eigenvalues $\lambda_{j}^{\prime}$ and $\lambda_{j}$. The proof that $T+A$ is a spectral operator depends on suitable estimates of these contour integrals, and is based on a new perturbation theorem due to $\mathrm{T}$. Kato [5].

Section 2 is devoted to perturbation theorems of a general nature, 
without reference to differential operators; the latter are treated in $\S 3$.

2. Relatively bounded perturbations. If $A$ is an arbitrary linear operator in the (complex) Hilbert space $\mathfrak{S}$, we denote by $\rho(A)$ the resolvent set of $A$, that is the set of all complex numbers $\lambda$ for which $R_{\lambda}(A)=(\lambda I-A)^{-1}$ exists as a bounded operator in $\mathfrak{S}$. The complement of $\rho(A)$ in the complex plane is the spectrum $\sigma(A)$. A closed operator $A$ in $\mathfrak{S}$ is called regular if for some $\lambda \in \rho(A)$, the resolvent operator $R_{\lambda}(A)$ is completely continuous. The spectrum of a regular operator consists of a sequence $\left\{\lambda_{n}\right\}$ of eigenvalues of finite multiplicity, having no accumulation point in the complex plane.

The definition of spectral operator is given for example in Schwartz [9], where the following result is proved [9, Lemma 3].

Lemma 1. Let $T$ be a regular spectral operator in the Hilbert space $\mathfrak{g}$. Assume that all but a finite number of the eigenvalues of $T$ are simple poles of the resolvent, and also that $\sum E\left(\lambda_{i}\right)=1$, where $E\left(\lambda_{i}\right)$ are the spectral projections of $T$. Then there exists a constant $c$ such that for any point $\lambda \in \rho(T)$ not in a fixed neighborhood of the exceptional multiple eigenvalues, we have

$$
\left\|R_{\lambda}(T)\right\| \leqq c[\operatorname{dist}(\lambda, \sigma(T))]^{-1} .
$$

Lemma 2. Let $T$ and $A$ be closed linear operators in $\mathfrak{S}$, with $\mathfrak{D}(A) \supset \mathfrak{D}(T)$, and suppose that $|A|_{T}=0$. Define the operator $T+A$, with $\mathfrak{D}(T+A)=\mathfrak{D}(T)$, by $(T+A) u=T u+A u$. Then $T+A$ is a closed operator, and moreover

(i) if $\lambda \in \rho(T) \cap \rho(T+A)$ then

$$
R_{\lambda}(T+A)-R_{\lambda}(T)=R_{\lambda}(T+A) \cdot A R_{\lambda}(T) ;
$$

(ii) if $\lambda \in \rho(T)$ and $\left\|A R_{\lambda}(T)\right\|<1$, then $\lambda \in \rho(T+A)$ and

$$
R_{\lambda}(T+A)-R_{\lambda}(T)=R_{\lambda}(T)\left[I-A R_{\lambda}(T)\right]^{-1} A R_{\lambda}(T) .
$$

The assertions of this lemma are easily verified. Note also that if $A$ is $T$-bounded then for $\lambda \in \rho(T), A R_{\lambda}(T)$ is a bounded operator in $\mathfrak{S}$ :

$$
\begin{aligned}
\left\|A R_{\lambda}(T) u\right\| & \leqq c_{1}\left\|(T+\lambda I-\lambda I) R_{\lambda}(T) u\right\|+c_{2}\left\|R_{\lambda}(T) u\right\| \\
& \leqq\left\{\left(c_{1}|\lambda|+c_{2}\right)\left\|R_{\lambda}(T)\right\|+c_{1}\right\}\|u\| \quad(u \in \mathfrak{S}) .
\end{aligned}
$$

TheoRem 1. Let $T$ be a regular spectral operator in $\mathfrak{S}$, and assume that its eigenvalues $\left\{\lambda_{n}\right\}$ satisfy

$$
\begin{aligned}
& \lambda_{n} \sim a n^{\alpha} \quad(n \rightarrow \infty), \\
& \lambda_{n+1}-\lambda_{n}=a(n) n^{\alpha-1},
\end{aligned}
$$


for some constants $a>0, \alpha>1$, where

$$
0<c_{1}<a(n)<c_{2} \quad(\text { large } n) .
$$

Assume also that $\sum E\left(\lambda_{i}\right)=1$.

Let $A$ be a closed operator in $\mathfrak{S}$, with $\mathfrak{D}(A) \supset \mathfrak{D}(T)$, having the following property: for each $\varepsilon, 0<\varepsilon<1$, there exists a real number $C_{\varepsilon}$ such that

$$
\|A u\| \leqq \varepsilon\|T u\|+C_{\varepsilon}\|u\|, \quad(u \in \mathfrak{D}(T))
$$

and

$$
C_{\varepsilon}=O\left(\varepsilon^{-\tau}\right) \quad \text { as } \quad \varepsilon \rightarrow 0^{+},
$$

for some number $\tau, 0 \leqq \tau \leqq \alpha-1$. For values of $n$ for which $\lambda_{n}>0$, let $\Gamma_{n}(\mu), \mu>0$, be the circle with centre $\lambda_{n}$ and radius $\mu \cdot \lambda_{n}^{\tau /(1+\tau)}$.

Then the operator $T+A$ (with $\mathfrak{D}(T+A)=\mathfrak{D}(T))$ is a closed regular operator in $\mathfrak{g}$. If $\tau<\alpha-1$ then for sufficiently large $\mu$, the eigenvalues $\lambda_{n}^{\prime}$ of $T+A$ can be enumerated so that $\lambda_{n}^{\prime}$ lies inside $\Gamma_{n}(\mu)$, with the possible exception of finitely many values of $n$. In case $\tau=\alpha-1$, there exists $\mu_{0}>0$ such that the same is true provided the constant involved in (2.7) is sufficiently small, i.e. provided

$$
\xi_{0}=\sup _{0<\varepsilon<1} \varepsilon^{\alpha-1} C_{\varepsilon}
$$

is sufficiently small.

Proof. We will consider the case in which $T$ is self-adjoint. The proof in the general case involves only slight modifications to cover the possibility of complex eigenvalues and non self-adjoint eigenprojections.

By Lemma 2, $T+A$ is closed. Since $T$ is regular, $R_{2}(T)$ is completely continuous for any $\lambda \in \rho(T)$. Identity (2.3) will then imply that $T+A$ is regular, provided we know that $\left\|A R_{\lambda}(T)\right\|<1$ for some $\lambda \in \rho(T)$. By (2.6) and (2.7) we have, for $u \in \mathfrak{T}, 0<\varepsilon<1$ and $\lambda \in \rho(T)$,

$$
\left\|A R_{\lambda}(T)\right\| \leqq\left(\varepsilon|\lambda|+C \varepsilon^{-\tau}\right)\left\|R_{\lambda}(T)\right\|+\varepsilon
$$

(cf. (2.4)). Choosing $\varepsilon$ so as to minimize the expression in parentheses, we obtain

$$
\begin{aligned}
& \left\|A R_{\lambda}(T)\right\| \leqq c_{1}|\lambda|^{-1 /(\tau+1)}+c_{2}|\lambda|^{\tau /(\tau+1)}\left\|R_{\lambda}(T)\right\| \\
& \quad(\lambda \in \rho(T),|\lambda|>c \tau) ;
\end{aligned}
$$

here the constants $c_{1}, c_{2}$ depend only on $\tau$; for $\tau=0$ we can take $c_{1}=0$. 
Since by Lemma $1,\left\|R_{\lambda}(T)\right\| \leqq(\operatorname{Im} \lambda)^{-1}$, we see that $\left\|A R_{\lambda}(T)\right\| \leqq$ const. $|\lambda|^{-1 /(\tau+1)}$ for purely imaginary $\lambda$, so that $\left\|A R_{\lambda}(T)\right\|<1$ for suitable $\lambda \in \rho(T)$. This ensures that $T+A$ is regular.

Consider now the case $\tau<\alpha-1$. Then $\lambda_{n}^{\tau /(1+\tau)}=o\left(n^{\alpha-1}\right)=$ $o\left(\min \left(\lambda_{n+1}-\lambda_{n}, \lambda_{n}-\lambda_{n-1}\right)\right)$. It follows that for any $\mu>0$, the circles $\Gamma_{n}(\mu)$ lie outside each other for $n \geqq N_{1}(\mu)$, and the only point of $\sigma(T)$ lying inside $\Gamma_{n}(\mu)$ is $\lambda_{n}$. Using (2.5), (2.8), and Lemma 1, we find that, for some $N(\mu) \geqq N_{1}(\mu)$,

$$
\left\|A R_{\lambda}(T)\right\| \leqq c_{1}|\lambda|^{-1 /(\tau+1)}+c_{2}^{\prime} \mu^{-1} \leqq c_{3} \mu^{-1} \quad\left(\lambda \in \Gamma_{n}(\mu), n \geqq N(\mu)\right) .
$$

Henceforth let $\mu$ satisfy

$$
c_{3} \mu^{-1} \leqq \frac{1}{3}
$$

Let $E\left(\lambda_{n}\right)$ denote the eigenprojection of $T$ corresponding to $\lambda_{n}$, and let $E_{n, \mu}^{\prime}$ denote the sum of the eigenprojections of $T+A$ corresponding to eigenvalues of $T+A$ lying inside $\Gamma_{n}(\mu)$. Since $\left\|A R_{\lambda}(T)\right\|<1$ on $\Gamma_{n}(\mu), n \geqq N(\mu)$, Lemma 2 (ii) shows that $T+A$ has no eigenvalues on $\Gamma_{n}(\mu)$, so that

$$
E_{n, \mu}^{\prime}-E\left(\lambda_{n}\right)=\frac{1}{2 \pi i} \int_{\Gamma_{n}(\mu)}\left[R_{\lambda}(T+A)-R_{\lambda}(T)\right] d \lambda .
$$

Hence by (2.1), (2.3) and (2.9),

$$
\left\|E_{n, \mu}^{\prime}-E\left(\lambda_{n}\right)\right\| \leqq \frac{c_{3} \mu^{-1}}{1-c_{3} \mu^{-1}} \leqq \frac{1}{2} .
$$

Therefore ([2, p. 587]) the ranges of $E_{n, \mu}^{\prime}$ and $E\left(\lambda_{n}\right)$ have the same dimension, namely 1 ; i.e. each circle $\Gamma_{n}(\mu), n \geqq N(\mu)$, contains one simple eigenvalue $\lambda_{n}^{\prime}$ of $T+A$.

Next we construct a contour $\Gamma_{0}$ containing the eigenvalues $\lambda_{1}, \lambda_{2}, \cdots, \lambda_{N-1}$ only, such that the integral of $\left\|R_{\lambda}(T+A)-R_{\lambda}(T)\right\|$ over $\Gamma_{0}$ is small provided $N \geqq N(\mu)$ is sufficiently large. This will show that $T+A$ has $N-1$ eigenvalues (counting possible multiplicities) inside $\Gamma_{0}$. Since also $R_{\lambda}(T+A)$ exists for $\lambda$ outside $\Gamma_{0}$ and all $\Gamma_{n}(\mu)$, $n \geqq N(\mu)$, the assertion of the theorem about the eigenvalues $\lambda_{n}^{\prime}$ will be established.

For $\Gamma_{0}$ we take the rectangle with sides formed by the lines $L_{1}: \operatorname{Re} \lambda=\zeta_{N}=(1 / 2)\left(\lambda_{N-1}+\lambda_{N}\right)$, some $N \geqq N(\mu) ; L_{2}: \operatorname{Re} \lambda=-\zeta_{0}<0$; $L_{3}: \operatorname{Im} \lambda=\eta_{0}>0 ; L_{4}: \operatorname{Im} \lambda=-\eta_{0}$. Consider first

$$
\begin{aligned}
\int_{L_{1}}\left\|R_{\lambda}(T+A)-R_{\lambda}(T)\right\| d \lambda \leqq & C \int_{-\infty}^{\infty}\left\{\frac{1}{\left(x^{2}+\zeta_{N}^{2}\right)^{1 /(\tau+1)}}\right. \\
& \left.+\frac{\left(x^{2}+\zeta_{N}^{2}\right)^{(1 / 2) \tau /(\tau+1)}}{\left(x^{2}+\delta_{N}^{2}\right)^{(1 / 2)}}\right\} \times \frac{d x}{\left(x^{2}+\delta_{N}^{2}\right)^{1 / 2}}
\end{aligned}
$$


where $\delta_{N}=(1 / 2)\left(\lambda_{N}-\lambda_{N-1}\right)$. The integral of the first term is easily estimated; the second does not exceed

$$
\zeta_{N}^{-1 /(\tau+1)} \int_{-\infty}^{\infty} \frac{\left(t^{2}+1\right)^{(1 / 2) \tau /(\tau+1)} d t}{t^{2}+\delta_{N}^{2} \cdot \zeta_{N}^{-2}} \leqq \zeta_{N}^{-1 /(\tau+1)} \int_{-\infty}^{\infty} \frac{\left(t^{2}+1\right)^{(1 / 2) \tau /(\tau+1)} d t}{t^{2}+c \cdot N^{-2}} .
$$

Treating separately the ranges $|t| \leqq 1$ and $|t|>1$ in the latter integral, we readily verify that its value is small for large $N$. As for the rest of $\Gamma_{0}$, simple calculations show, for suitable choices of $\zeta_{0}, \eta_{0}$, first that the contribution of $L_{2}$ is small, and then that the contribution from the sections $L_{3}, L_{4}$ lying between $L_{1}$ and $L_{2}$ is also small. Thus $\Gamma_{0}$ has the required property.

For the case $\tau=\alpha-1$, notice that the constants $c_{1}, c_{2}$ in (2.8) are small provided $\xi_{0}$ is small. Thus this case can be dealt with in the same way as above, and the proof is complete.

For our next result, the hypotheses about the perturbation $A$ are of a slightly different nature than in Theorem 1 . We will suppose that $A=B T^{(\alpha-1) / \alpha}$ where $B=B_{1}+B_{2}$, the sum of a bounded operator $B_{1}$ of sufficiently small norm, and a compact operator $B_{2}$. Perturbations of this sort have been considered by Turner [11]. From Lemma 3 below we see that such an operator $A$ is $T$-bounded, and satisfies (2.6) and (2.7), with $\tau=\alpha-1$.

The operator $T^{\theta}$ ( $\theta$ real) is defined by means of the functional calculus. Suppose $T$ is a spectral operator with spectral family $\left\{E_{j}\right\}$, such that $E_{j}$ is one-dimensional for $j \geqq 1$, and $E_{0}=\sum_{0}^{k} E_{0 i}$, each $E_{0 i}$ being a finite dimensional projection corresponding to an eigenvalue $\lambda_{0 i}$. If $f$ is a sufficiently smooth function which is uniformly bounded on the spectrum $\sigma(T)$, then $f(T)$ is defined by the formula (cf. [9])

$$
f(T)=\sum_{i=0}^{k} \sum_{m=0}^{\mu_{i}} \frac{f^{(m)}\left(\lambda_{0 i}\right)}{m !}\left(T-\lambda_{0 i}\right)^{m} E_{0_{i}}+\sum_{j=1}^{\infty} f\left(\lambda_{j}\right) E_{j}
$$

where $\mu_{i}$ is the algebraic multiplicity of $\lambda_{0 i}$. In this expression, the first sum, being finite dimensional, plays a rather trivial role in analytic arguments, and we will generally omit details. The following is derived by a simple calculation.

Lemma 3. Let $T$ satisfy the above conditions, and let $0 \leqq \theta \leqq 1$. Then there exists a constant $C=C(\theta)$ such that

$$
\left\|T^{\theta} u\right\| \leqq \varepsilon\|T u\|+C \varepsilon^{-\theta /(1-\theta)}\|u\|
$$

for all $u \in \mathfrak{D}\left(T^{\theta}\right)$ and $0<\varepsilon \leqq 1$.

We also require the following recent result of Kato [5] concerning 
perturbation of spectral families. By a $p$-sequence we mean a sequence $\left\{P_{j}\right\}$ of (not necessarily self-adjoint) projections in a Hilbert space $\mathfrak{S}$, satisfying the orthogonality conditions

$$
P_{j} P_{k}=\delta_{j k} \quad(j, k \geqq 0) .
$$

A $p$-sequence $\left\{E_{j}\right\}$ is self-adjoint if $E_{j}^{*}=E_{j}$ for all $j$. A self-adjoint $p$-sequence is complete if $\sum E_{j}=I$.

Lemma 4 (Kato). Let $\left\{P_{j}\right\}$ be a p-sequence and $\left\{E_{j}\right\}$ a complete self-adjoint p-sequence. Assume that

(i) $\operatorname{dim} P_{0}=\operatorname{dim} E_{0}=m<\infty$,

(ii) $\sum_{j=1}^{\infty}\left\|E_{j}\left(P_{j}-E_{j}\right) u\right\|^{2} \leqq c^{2}\|u\|^{2}$

for all $u \in \mathfrak{S}$, where $c$ is a constant, $0 \leqq c<1$. Then $\left\{P_{j}\right\}$ is similar to $\left\{E_{j}\right\}$, i.e. there exists a nonsingular linear operator $W$ such that for all $j \geqq 0, P_{j}=W^{-1} E_{j} W$.

The proof of this lemma is fairly simple: set $W=\sum_{j=0}^{\infty} E_{j} P_{j}$; one shows that $W$ is well-defined and bounded, and using standard theorems about the index, that nullity $W=$ defect $W=0$. We refer to [5] for details.

THEOREM 2. Let $T$ be a regular spectral operator in $\mathfrak{S}$, and suppose the eigenvalues of $T$ satisfy the hypotheses (2.5) of Theorem 1. Let $A=\left(B_{1}+B_{2}\right) T^{(\alpha-1) / \alpha}$ where $B_{1}$ is a bounded operator in $\mathfrak{S}$, of sufficiently small norm, and $B_{2}$ is a compact operator. Then $T+A$ is a regular spectral operator; moreover the eigenvalues $\left\{\lambda_{n}^{\prime}\right\}$ of $T+A$ can be enumerated so that $\lambda_{n}^{\prime}$ lies inside the circle $\Gamma_{n}(\mu)$ (defined in Theorem 1) for large $n$.

Proof. Expressing $A R_{\lambda}(T)$ by means of the functional calculus, we obtain

$$
A R_{\lambda}(T)=B(\lambda)+\left(B_{1}+B_{2}\right) \sum_{j=1}^{\infty} \frac{\lambda_{i}^{(\alpha-1) / \alpha}}{\lambda_{j}-\lambda} E\left(\lambda_{j}\right),
$$

where $\|B(\lambda)\|=O\left(|\lambda|^{-1}\right.$ ) as $\lambda \rightarrow \infty$. (We are assuming, without loss of generality, that no $\lambda_{j}$ vanishes.) We will express the sum in two parts, $\sum_{1}^{p}+\sum_{p+1}^{\infty}$. In the second of these, we can replace $\left(B_{1}+B_{2}\right)$ by $\left(B_{1}+B_{2}\right) \widetilde{E}_{p}$ where $\widetilde{E}_{p}=\sum_{p+1}^{\infty} E\left(\lambda_{j}\right)$. Since $B_{2}$ is a compact operator we have $\left\|B_{2} \widetilde{E}_{p}\right\|=\varepsilon_{p} \rightarrow 0$ as $p \rightarrow \infty$. The sum $\sum_{1}^{p}$ can be combined with $B(\lambda)$, and we reach the following estimate:

$$
\begin{aligned}
\left\|A R_{\lambda}(T)\right\|^{2} \leqq & c\left(\left\|B_{1}\right\|+\varepsilon_{p}\right)^{2} \sum_{j=p+1}^{\infty} \frac{\left|\lambda_{j}\right|^{2(\alpha-1) / \alpha}\left\|E\left(\lambda_{j}\right)\right\|^{2}}{\left|\lambda_{j}-\lambda\right|^{2}} \\
& +C_{p}|\lambda|^{-2} .
\end{aligned}
$$


For $\lambda \in \Gamma_{n}(\mu)$, the sum in (2.11) is bounded independently of $p$ (a more detailed estimate for this sum appears below). Hence with $\left\|B_{1}\right\|+\varepsilon_{p}$ sufficiently small, we can choose $N$ so that $\left\|A R_{\lambda}(T)\right\| \leqq \delta<1$ for $\lambda \in \Gamma_{n}(\mu), n \geqq N$. By (2.3) this implies that $\left\|R_{\lambda}(T+A)\right\|<$ const. $r_{n}^{-1}$. Therefore (with the notation of Theorem 1 ) we have

$$
\begin{aligned}
\left\|\left(E_{n, \mu}^{\prime}-E\left(\lambda_{n}\right)\right) u\right\| & =\left\|\frac{1}{2 \pi i} \int_{\Gamma_{n}(\mu)} R_{\lambda}(T+A)\left[I-A R_{\lambda}(T)\right]^{-1} A R_{\lambda}(T) u d \lambda\right\| \\
& \leqq c \sup _{\lambda \in \Gamma_{n}(\mu)}\left\|A R_{\lambda}(T) u\right\| \leqq \frac{1}{2}\|u\|
\end{aligned}
$$

provided $\left\|B_{1}\right\|$ is sufficiently small and $n$ sufficiently large. This proves the assertion about the eigenvalues $\lambda_{n}^{\prime}$.

We pass now to the proof that $T+A$ is spectral. If $E_{0}, E\left(\lambda_{1}\right)$, $E\left(\lambda_{2}\right), \cdots$ are the spectral projections for $T\left(E\left(\lambda_{i}\right)\right.$ being one-dimensional), then according to the theorem of Lorch-Mackey-Wermer [12], this family is similar to a complete self-adjoint $p$-sequence $\left\{E_{j}\right\}$. There is no loss of generality in supposing the similarity to be the identity transformation. By taking $\operatorname{dim} E_{0}$ large enough we may also suppose that the circles $C_{n}=\Gamma_{n}(\mu), n>0$, are separated, and that their radii satisfy $r_{n} \geqq c \cdot n^{\alpha-1}$ (with $c>0$ ).

Let $P_{n}$ denote the eigenprojection of $T+A$ corresponding to $\lambda_{n}^{\prime}$. We wish to verify that the hypotheses of Kato's lemma are satisfied. First we can show that $\operatorname{dim} E_{0}=\operatorname{dim} P_{0}$ provided sufficiently many of the eigenprojections $E_{j}$ are included in $E_{0}$. The proof is the same as in Theorem 1, modified to utilize the compactness of $B_{2}$ in the same way as above.

Next, it is obviously sufficient to show that for some integer $N$ we have

$$
\sum_{n=N}^{\infty}\left\|E_{n}\left(P_{n}-E_{n}\right) u\right\|^{2} \leqq c^{2}\|u\|^{2} \quad\left(c^{2}<1\right) .
$$

Using (2.11) we have for any integer $p>1$

$$
\begin{aligned}
\sum_{n=. N}^{\infty} \| & E_{n}\left(P_{n}-E_{n}\right) u \|^{2} \\
\leqq & c \sum_{n=N}^{\infty} \sup _{\lambda \in C_{n}}\left(\left\|B_{p}(\lambda) u\right\|^{2}+\left(\left\|B_{1}\right\|+\varepsilon_{p}\right)^{2}\right. \\
& \left.\cdot \sum_{k=p+1}^{\infty}\left|\lambda_{k}\right|^{2(\alpha-1) / \alpha}\left|\lambda_{k}-\lambda\right|^{-2}\left\|E_{k} u\right\|^{2}\right) \\
\leqq & c_{p}\left(\sum_{n=N}^{\infty}\left|\lambda_{n}\right|^{-2}\right)\|u\|^{2} \\
& +c^{\prime}\left(\left\|B_{1}\right\|+\varepsilon_{p}\right)^{2}\left[\sum_{n=N}^{\infty} \sum_{p+1 \leqq k \neq n}\left|\lambda_{k}\right|^{2-2 / \alpha}\left|\lambda_{k}-\lambda_{n}\right|^{-2}\left\|E_{k} u\right\|^{2}\right. \\
& \left.+\sum_{n=N}^{\infty} r_{n}^{-2}\left|\lambda_{n}\right|^{2-2 / \alpha}\left\|E_{n} u\right\|^{2}\right] .
\end{aligned}
$$


The three sums here (from $N$ to $\infty$ ) are fairly easily estimated. Assume that $p$ has been chosen, and $\left\|B_{1}\right\|+\varepsilon_{p}$ is suitably small. Since $\lambda_{k} \sim a k^{\alpha}$, the first sum in square brackets can be approximated by

$$
\text { const. }\left\{\sum_{k=1}^{\infty} k^{-2}\left[\sum_{1 \leqq n \neq k}\left|1-(n / k)^{\alpha}\right|^{-2}\right] \cdot\left\|E_{k} u\right\|^{2}\right\} \leqq \text { const. } \sum_{k=1}^{\infty}\left\|E_{k} u\right\|^{2} \text {, }
$$

because by an elementary calculation, the sum in the square brackets here is $O\left(k^{2}\right)$. Since the first and last sums above are trivial to estimate, we finally obtain

$$
\sum_{n=N}^{\infty}\left\|E_{n}\left(P_{n}-E_{n}\right) u\right\|^{2} \leqq c^{2}\|u\|^{2}
$$

where $c^{2}<1$ provided $\left\|B_{1}\right\|$ is small and $N$ large. This completes the proof.

Corollary. Suppose that $A$ and $T$ satisfy the hypotheses of Theorem 1, and that $\tau<\alpha-1$. Then $T+A$ is a spectral operator.

Proof. It follows from (2.6) and (2.7) that

$$
\|A u\| \leqq C\|T u\|^{\tau /(\tau+1)}\|u\|^{1 /(\tau+1)}, \quad u \in \mathscr{D}(T) .
$$

If we assume, as we may without loss of generality, that $\sigma(T)$ lies entirely in the open right half-plane, we can apply a theorem of Krasnoselsky and Sobolevsky [7, Th. 5] to conclude that $A T^{-\sigma}$ is a bounded operator, for any $\sigma>\tau /(\tau+1)$. In particular, we can choose $\sigma$ such that $\tau /(\tau+1)<\sigma<(\alpha-1) / \alpha$, and write

$$
A=B T^{(\alpha-1) / \alpha} \quad \text { with } B=\left(A T^{-\sigma}\right)\left(T^{\sigma-l(\alpha-1) / \alpha}\right) .
$$

Since $T^{\mu}$ is compact for any $\mu<0$ (see [7]), we see that $B$ is a compact operator. It follows from the Theorem, therefore, that $T+A$ is spectral.

REMARK. If $\tau<\alpha-1$ is given, the proof of Theorem 1 will yield explicit constants $C(\tau)$ and $N(\tau)$ such that

$$
\left|\lambda_{n}^{\prime}-\lambda_{n}\right|<C(\tau)\left|\lambda_{n}\right|^{\tau /(\tau+1)}
$$

for $n \geqq N(\tau)$. The same information cannot be derived via the above Corollary, since $\left\|A T^{-\sigma}\right\|$ may approach infinity in an unspecified fashion as $\sigma \rightarrow \tau /(\tau+1)^{+}$. The case $\tau=\alpha-1$ is, of course, not covered at all by the Corollary.

3. Application. Let $I=\left[x_{0}, x_{1}\right]$ be a finite closed interval, $x_{0}<x_{1}$, and consider the Sobolev space $H^{m}(I)$ consisting of all $f \in L_{2}(I)$ having generalized derivatives $D^{j} f$ also in $L_{2}(I)$, for $j \leqq m$. The norm 
in $H^{m}(I)$ is given by

$$
\|f\|_{m}=\left\{\sum_{j=0}^{m} \int_{I}\left|D^{j} f(x)\right|^{2} d x\right\}^{1 / 2} .
$$

We denote by $H_{0}^{m}(I)$ the closure in $H^{m}(I)$ of $C_{0}^{\infty}\left(I^{0}\right)$, the space of infinitely differentiable functions whose support is a compact subset of the open interval $\left(x_{0}, x_{1}\right)$. If $W$ is any closed subspace such that

$$
H_{0}^{2 m}(I) \subset W \subset H^{2 m}(I),
$$

we define an operator $T_{W}$ in $\mathfrak{g}=L_{2}(I)$ by

$$
\begin{aligned}
\mathfrak{D}\left(T_{W}\right) & =W \\
T_{W} f & =(-1)^{m} D^{2 m} f .
\end{aligned}
$$

Explicit forms of boundary conditions determining $W$ have been studied extensively, cf. [2, Ch. XIII]. In particular, it is known that under quite general conditions $T_{W}$ is a regular spectral operator, with eigenvalues satisfying (2.5) for $\alpha=2 m$; see [2], [6], and [8] for details.

The perturbing operator $A$ is now defined as the closure of the operator $A_{0}$ :

$$
\begin{aligned}
\mathfrak{D}\left(A_{0}\right) & =W \\
A_{0} f & =\sum_{k=0}^{2 m-1} Q_{k}\left(D^{k} f\right),
\end{aligned}
$$

the $Q_{k}$ denoting arbitrary bounded operators in $\mathfrak{S}$.

Lemma 5. Let $j, k$ be nonnegative integers, $j<k, k \geqq 2$. Then there exists a constant $C=C_{j k}$ such that for all $\varepsilon, 0<\varepsilon<1$, and all $f \in H^{k}(I)$,

$$
\begin{aligned}
& \left\{\int_{I}\left|D^{j} f(x)\right|^{2} d x\right\}^{1 / 2} \\
& \quad \leqq \varepsilon\left\{\int_{I}\left|D^{k} f(x)\right|^{2} d x\right\}^{1 / 2}+C \varepsilon^{-j /(k-j)}\left\{\int_{I}|f(x)|^{2} d x\right\}^{1 / 2} .
\end{aligned}
$$

This result can be proved by elementary but tedious calculations; a complete proof (in $n$ dimensions) is given in [1, pp. 17-25]. The following is obvious.

Corollary. There exists a constant $C$, independent of the operators $Q_{k}$, such that for $0<\varepsilon_{i}<1(i=1,2, \cdots, 2 m-1)$ and $f \in W$,

$$
\begin{aligned}
\|A f\| \leqq & \left(\sum_{k=0}^{2 m-1} \varepsilon_{k}\left\|Q_{k}\right\|\right)\|T f\|_{0} \\
& +C\left(\sum_{k=0}^{2 m-1}\left\|Q_{k}\right\| \varepsilon_{k}^{-k /(2 m-k)}\right)\|f\|_{0} .
\end{aligned}
$$


Theorem 3. Let $T_{W}$ and $A$ be given by (3.1) and (3.2) respectively, and assume that $T_{W}$ is a spectral operator, with eigenvalues $\left\{\lambda_{n}\right\}$ satisfying (2.5). Let $\left\{\lambda_{n}^{\prime}\right\}$ be the eigenvalues of the regular operator $T_{W}+A$. Assume that $Q_{2 m-1}=B_{1}+B_{2}$ where $\left\|B_{1}\right\|$ is sufficiently small and $B_{2}$ is a compact operator, and that the remaining coefficients $Q_{j}$ are bounded operators. Then for large $n$,

$$
\left|\lambda_{n}^{\prime}-\lambda_{n}\right| \leqq c\left|\lambda_{n}\right|^{k / 2 m},
$$

where $k$ is defined by (1.6). Moreover $T_{W}+A$ is a spectral operator.

Proof. Suppose first that $k \leqq 2 m-2$. Letting $\varepsilon_{0}=\varepsilon_{1}=\cdots=$ $\varepsilon<1$ in (3.4) we obtain

$$
\|A f\| \leqq c_{1} \varepsilon\|T f\|+c_{2} \varepsilon^{-k /(2 m-k)}\|f\|
$$

for $f \in \mathfrak{D}\left(T_{W}\right)$. Hence the hypotheses of Theorem 1 are satisfied, with $\tau=k /(2 m-k)$, i.e. $\tau+1 \leqq m=\alpha / 2 \leqq \alpha-1$. Hence the results in this case are immediate consequences of Theorem 1 and the Corollary to Theorem 2.

For the case $k=2 m-1$, let us write $A_{0}=Q_{2 m-1} D^{2 m-1}$ and $A=$ $A_{0}+A_{1}$. By the first part of the proof, $T_{W}+A_{1}$ is a spectral operator with eigenvalues $\left\{\lambda_{n 1}\right\}$ satisfying (3.5) for $k=2 m-2$. The eigenvalues $\left\{\lambda_{n 1}\right\}$ therefore satisfy the hypotheses (2.5) of Theorem 1 .

Now we can write $A_{0}=\left(B_{1}^{\prime}+B_{2}^{\prime}\right) T^{(2 m-1) / 2 m}$, where

$$
B_{i}^{\prime}=B_{i} D^{2 m-1} T^{-(2 m-1) / 2 m} .
$$

Since $T^{-(2 m-1) / 2 m}$ is a continuous linear map from $L_{2}(I)$ to $H^{2 m-1}(I)$ (cf. [2, Ch. XIII]) and $D^{2 m-1}$ is continuous from $H^{2 m-1}(I)$ to $L_{2}(I)$, we see that $B_{1}^{\prime}$ is a bounded operator in $L_{2}(I)$ with $\left\|B_{1}^{\prime}\right\| \leqq c\left\|B_{1}\right\|$; also $B_{2}^{\prime}$ is compact. An application of Theorem 2 to the operator $T_{W}+A=$ $\left(T_{W}+A_{1}\right)+A_{0}$ then yields the desired conclusions, and the proof is complete.

The author wishes to thank Professor Tosio Kato for several valuable suggestions. The general idea of the proof of Theorem 2 is due to him.

\section{REFERENCES}

1. S. Agmon, Lectures on Elliptic Boundary Value Problems, Van Nostrand, Princeton, 1965.

2. N. Dunford and J. Schwartz, Linear Operators I; II; III, Interscience, New York, 1958; 1963; in press.

3. K. O. Friedrichs, On the perturbation of continuous spectra, Comm. Pure Appl. Math. 1 (1948), 361-406.

4. T. Kato, Perturbation theory of linear operators, Grundlehren der Math. Wiss. 132, Springer-Verlag. Heidelberg, 1966. 
5. - Similarity for sequences of projections, Bull. Amer. Math. Soc. 73 (1967), 904-905.

6. H. P. Kramer, Perturbation of differential operators, Pacific J. Math. 7 (1957), 1405-1435.

7. M. A. Krasnoselski and P. E. Sobolevski, Fractional powers of operators defined in Banach spaces, Dokl. Akad. Nauk SSSR 129 (1959), 499-502.

8. M. A. Naimark, Linear Differential Operators, Gosud. Izdat. Tek.-Teor. Lit., Moscow, 1954.

9. J. Schwartz, Perturbations of spectral operators, and applications, I. Bounded perturbations, Pacific J. Math. 4 (1954), 415-458.

10. A. E. Taylor, Introduction to Functional Analysis, Wiley, New York, 1958.

11. R. E. L. Turner, Perturbation of ordinary differential operators, Journal of Math. Anal. Appl. 13 (1966), 447-457.

12. J. Wermer, Commuting spectral measures on Hilbert space, Pacific J. Math. 4 (1954), 355-361.

Received November 29, 1966. Research sponsored by the Air Force Office of Scientific Research, Office of Aerospace Research, United States Air Force, under AFOSR Grant Nr. AF-AFOSR 379-65.

The University of British Columbia 


\section{PACIFIC JOURNAL OF MATHEMATICS}

\section{EDITORS}

H. ROYDEN

Stanford University

Stanford, California

\section{J. P. Jans}

University of Washington

Seattle, Washington 98105

\section{J. DUGUNDJI}

Department of Mathematics University of Southern California Los Angeles, California 90007

\section{RICHARD ARENS}

University of California

Los Angeles, California 90024

\section{ASSOCIATE EDITORS}

E. F. BECKENBACH

B. H. NEUManN

F. WOLF

K. YosIDA

\section{SUPPORTING INSTITUTIONS}

UNIVERSITY OF BRITISH COLUMBIA CALIFORNIA INSTITUTE OF TECHNOLOGY UNIVERSITY OF CALIFORNIA MONTANA STATE UNIVERSITY UNIVERSITY OF NEVADA NEW MEXICO STATE UNIVERSITY OREGON STATE UNIVERSITY UNIVERSITY OF OREGON OSAKA UNIVERSITY UNIVERSITY OF SOUTHERN CALIFORNIA
STANFORD UNIVERSITY

UNIVERSITY OF TOKYO UNIVERSITY OF UTAH WASHINGTON STATE UNIVERSITY UNIVERSITY OF WASHINGTON AMERICAN MATHEMATICAL SOCIETY CHEVRON RESEARCH CORPORATION TRW SYSTEMS NAVAL WEAPONS CENTER 


\section{Pacific Journal of Mathematics}

\section{Vol. 25, No. $1 \quad$ September, 1968}

Glen Eugene Bredon, Cosheaves and homology................... 1

Robin Ward Chaney, A chain rule for the transformation of integrals in

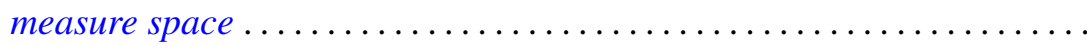

Colin W. Clark, On relatively bounded perturbations of ordinary differential operators................................... 59

John Edwin Diem, A radical for lattice-ordered rings.............. 71

Zeev Ditzian, On a class of convolution transforms ................ 83

Dennis Garoutte and Paul Adrian Nickel, A note on extremal properties characterizing weakly $\lambda$-valent principal functions............. 109

Shwu-Yeng Tzeng Lin, Fixed point properties and inverse limit spaces . . . 117

John S. Lowndes, Some dual series equations involving Laguerre polynomials ................................. 123

Kirti K. Oberai, Sum and product of commuting spectral operators ....... 129

J. N. Pandey and Armen H. Zemanian, Complex inversion for the generalized convolution transformation..................... 147

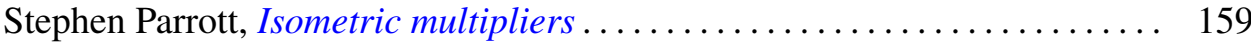

Manoranjan Prasad, Note on an extreme form .................. 167

Maciej Skwarczyński, A representation of a bounded function as infinite product in a domain with Bergman-Shilov boundary surface ......... 177

John C. Taylor, The Šilov boundary for a lattice-ordered semigroup ...... 185 Donald Reginald Traylor and James Newton Younglove, On normality and pointwise paracompactness ............................. 193

L. Tzafriri, Quasi-similarity for spectral operators on Banach spaces ... 